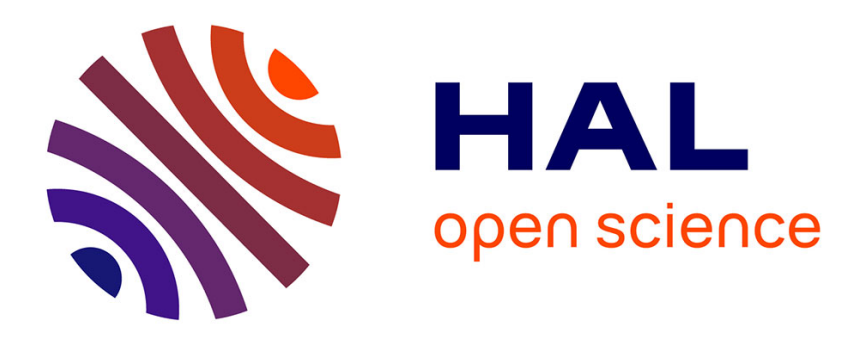

\title{
Histoire des populations noires ou histoire des rapports sociaux de race
}

\author{
Rygiel Philippe
}

\section{To cite this version:}

Rygiel Philippe. Histoire des populations noires ou histoire des rapports sociaux de race. Le Mouvement social, 2006, 215, pp.81-86. halshs-00655613

\section{HAL Id: halshs-00655613 https://shs.hal.science/halshs-00655613}

Submitted on 3 Jan 2012

HAL is a multi-disciplinary open access archive for the deposit and dissemination of scientific research documents, whether they are published or not. The documents may come from teaching and research institutions in France or abroad, or from public or private research centers.
L'archive ouverte pluridisciplinaire HAL, est destinée au dépôt et à la diffusion de documents scientifiques de niveau recherche, publiés ou non, émanant des établissements d'enseignement et de recherche français ou étrangers, des laboratoires publics ou privés. 


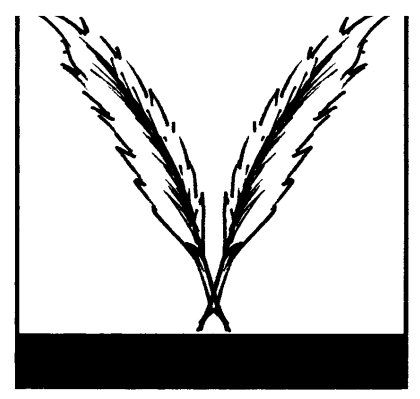

\section{CONTROVERSE}

\section{HISTOIRE DES POPULATIONS NOIRES OU HISTOIRE DES RAPPORTS SOCIAUX DE RACE par Philippe RYGIEL*}

"Le poème transforme en résultat ce qui l'avait précédé " César Aria, Varama

Pap Ndiaye a récemment présenté aux lecteurs du Mouvement Social les préalables théoriques d'une histoire des populations noires en France (1). Invité à discuter ce texte, je propose ici quelques remarques qui, c'est la loi du genre, s'attachent surtout à ce qui dans son entreprise pose selon moi problème, même s'il me faut indiquer que je partage certaines de ses positions. Je considère ainsi que les races ont un sens, dans le contexte français, en tant que tropes idéologiques (2) et catégories de perception (3), et nous pouvons repérer des rapports sociaux (4), des pratiques administratives (5) ou des décisions politiques (6) qui incorporent manifestement des schèmes raciaux, c'est-à-dire, puisqu'il nous faut bien une définition générique, malgré l'extrême diversité des discours (7), affirmant que les individus présentent des traits comportementaux et moraux déterminés par leur origine raciale et/ou ethnique. Ce n'est pas là un phénomène récent. Au début du XXe siècle, la plupart des responsables politiques français intervenant dans la définition des

* Maitre de conférences d'histoire contemporaine à l'Université Paris I.

(1) P. NDIAYE, "Pour une histoire des populations noires en France : préalables théoriques ", Le Mouvement Social, octobre-décembre 2005, p. 91-108.

(2) Par exemple, P.-A. TAGUiefF, La couleur et le sang. Doctrines racistes à la française, Paris, Éditions Mille et une nuits, 1998.

(3) A. GIRARD, Y. CharBit, M.-L. LAmY, "Attitudes des Français à l'égard de l'immigration étrangère, nouvelle enquête d'opinion ", Population, novembre-décembre 1974, p. 1015-1069.

(4) P. BATAILle, Le racisme au travail, Paris, La Découverte, 1997.

(5) A. SPIRE, Étrangers à la carte. L'administration de l'immigration en France, 1945-1975, Paris, Grasset, 2005.

(6) J.-L. AmSELle, Vers un multiculturalisme français. L'empire de la coutume, $2^{e}$ éd., Paris, Flammarion, 2001.

(7) P.-A. TAGUIEFF, "Face à l'immigration : mixophobie, xénophobie ou sélection. Un débat français dans l'entre-deux-guerres ", Vingtième Siècle, juillet-septembre 1995.

Le Mouvement Social, $n^{0} 215$, avril-juin 2006, @ Les Éditions de l'Atelier/Éditions Ouvrières 
politiques d'immigration envisageaient la mise en place d'une sélection raciale (8). De même, Louis Chevalier avait montré à quel point les observateurs sociaux de la monarchie censitaire empruntaient volontiers au registre de la race pour décrire les migrants provinciaux assiégeant Paris (9). Une histoire des usages politiques des catégories raciales et des rapports sociaux incorporant une référence raciale est donc possible dans le contexte français et, si nous disposons de travaux permettant de l'ébaucher (10), elle demeure à ce jour très lacunaire.

Il me semble qu'il suffit, pour justifier une telle histoire ou comprendre son émergence, de constater que de nombreux acteurs sont à l'heure actuelle engagés dans la construction d'identités collectives qui, quoique parfois concurrentes, ont en commun de viser à regrouper des individus et des groupes pris dans des rapports de domination socioethniques (11), dont les effets les plus apparents sont l'institutionnalisation de mécanismes discriminatoires. Faire la généalogie d'un mécanisme de domination ou d'un acteur collectif émergent, où dont on souhaite l'émergence, est une méthode éprouvée et efficace.

Je considère par contre que présenter la préparation d'une " histoire des populations noires de France" comme le moyen de réparer un oubli historiographique surprenant et à la limite scandaleux, comparaison avec la situation américaine à l'appui, ne peut que rendre plus difficile la défense de tels projets et illustrer la difficulté à mener à bien une enquête qui importe des catégories et des formes de mise en récit élaborées dans d'autres contextes. Chercher ainsi des explications complexes au fait que les historiens de la France se soient peu penchés sur la question noire alors que les historiens américains ou les spécialistes français des États-Unis sont nombreux à étudier Noirs et Amérindiens me paraît conduire à la production d'énoncés très contestables. Les actes fondateurs de la nation française n'en font pas un club d'hommes blancs, dont la supériorité raciale justifie la domination et l'accaparement des terres. La question noire n'a pas non plus été mêlée à une violente guerre civile mettant en jeu l'existence même de la nation, et les équilibres politiques locaux et nationaux n'ont jamais été remis en cause par l'émergence d'une question noire. Bref, celle-ci n'est pas intimement liée au roman des origines de la nation et elle n'a jamais, à ce jour, constitué une question politique centrale, ce qui me semble suffire à expliquer l'écart constaté.

De même, il me semble que les problèmes que me pose la définition de son objet par Pap Ndiaye sont, pour partie, imputables aux difficultés de l'usage, dans le contexte français, et dans une perspective rétrospective, de pratiques et de catégories élaborées par la sociologie américaine. Pap Ndiaye pose ainsi qu'il est possible, dans la France contemporaine, de définir un groupe social réunissant des individus

(8) C. Rosenberg, "Albert Sarraut and Republican Racial Thought ", French Politics, Culture and Society, Fall 2002, p. 97-114. Pour un exemple A. THOMAS, "Préface" à M. PAON, L'immigration en France, Paris, Payot, 1926, p. 12-13.

(9) L. Chevalier, Classes laborieuses et classes dangereuses à Paris dans la première moitié du XIXe siècle (1958), $2^{e}$ éd., Paris, Plon, 1970.

(10) H. Chapman, L. Frader (eds.), Race in France. Interdisciplinary Perspectives on the Politics of Difference, New York-Oxford, Berghahn Books, 2004.

(11) "La politique républicaine de l'identité ", Mouvements, mars 2005 
partageant " une même expérience sociale " au principe d'une "identité noire ", dont l'existence est affirmée. Que l'on puisse dans la France d'aujourd'hui se voir refuser un emploi ou un logement du fait de la couleur de sa peau est un fait (12). Nul ne conteste non plus le fait que les sociologues puissent construire et étudier des groupes dont les membres déclarent une même identité, ou furent placés dans de communes circonstances sociales, ni d'ailleurs que plusieurs modalités d'une identité noire ne soient aujourd'hui repérables en France, et qu'il soit possible de faire l'histoire de leur construction.

Je conteste cependant, ce qui me semble une conséquence des propos de Pap Ndiaye, que le groupe ainsi défini puisse être considéré comme un groupe social, au sens fort que son texte donne implicitement à cette expression - sans même poser la question de ce qu'implique en soi l'opération qui consiste à nommer groupe social la collection d'individus construite par le sociologue - et plus encore qu'il soit possible de déduire de l'existence actuelle du groupe son existence passée. Si nous suivons son texte, il faut pour cela qu'au moins trois conditions soient réunies. L'appartenance au groupe doit posséder une forte valeur explicative, quand sont examinées pratiques ou positions des membres du groupe. Ses membres doivent avoir conscience de cette identité partagée, ce qui suppose, pour que l'observateur puisse le vérifier, qu'elle soit parfois exprimée. Enfin l'expérience sociale partagée doit être spécifique aux membres du groupe. Cela n'est pas sans poser problèmes. Pour n'évoquer que les dernières décennies (13), les migrants provenant d'Afrique subsaharienne et les Antillais présents en métropole durant le dernier demi-siècle se sont heurtés à des discriminations. Celles-ci n'étaient pas toutes de même nature. Si les uns subirent des discriminations socio-raciales, les autres furent victimes également de discriminations légales visant les étrangers et il n'est pas a priori certain, du fait en particulier des effets sociaux des catégories juridiques, que les formes de l'insertion sociale des populations en provenance d'Afrique subsaharienne n'aient pas ressemblé plus à celles d'autres populations étrangères qu'à celles des Antillais.

De même, il n'est pas certain que l'expérience partagée de discriminations socioraciales suffise à définir une identité commune, du moins si nous parlons, ce qui semble être le cas de Pap Ndiaye, d'une identité fondant des prises de position publiques et non d'une identité dormante. David Beris, étudiant les Antillais présents en métropole, notait récemment que ceux-ci préféraient très généralement défendre une identité antillaise plutôt que participer à la construction d'une identité noire (14).

Je conteste de plus, quand bien même nous pourrions établir l'existence d'un groupe possédant de tels attributs dans le contexte de la France contemporaine, qu'il

(12) M. ReBZANI, Des jeunes dans la discrimination, Paris, PUF, 2002.

(13) J. Barou, Travailleurs africains en France, Grenoble, POF-PUG, 1978. C. QuiminaL, Gens d'ici, gens d'ailleurs. Migrations soninké et transformations villageoises, Paris, Bourgois, 1991.

(14) D. BerIS, Black Skins, French Voices. Caribbean Ethnicity And Activism In Urban France, Boulder, Westview, 2004. De même, étudiant le personnel du milieu hospitalier parisien, Marguerite Cognet a récemment mis en évidence de fortes similitudes dans les trajectoires identitaires des personnels en provenance du Quercy et des Antilles et le refus de la plupart de ceux-ci d'être associés aux "Africains ". M. COGNET, "Trajectoire de la différence des groupes ethnicisés. Des Auvergnats aux Antillais ", Revue européenne des migrations internationales, avril-juin 1999, p. 167-187. 
s'en déduise la possibilité d'une histoire des populations noires de France, définies comme telles, en partie sans doute parce que, comme j'ai produit quelques travaux de démographie historique, le terme de population, accolé à un mot évoquant l'origine, a pour moi un sens fort et précis et renvoie tant à un imaginaire puissant, qui est celui de la race et de la nation (15), qu'à un ensemble de techniques précisément définies. Celles-ci supposent en particulier que l'on puisse identifier des individus en tant que Noirs et qu'il sera ensuite possible de les dénombrer, opération qui n'a pas le même sens dans le contexte américain et dans le contexte français. La solidité de la catégorie noire dans le contexte américain ne provient pas en effet seulement du partage d'une expérience sociale mais du fait que "blanc" - et donc "non blanc" est durant une bonne partie de la période contemporaine une catégorie juridique, au sens où des droits spécifiques sont subordonnés à l'appartenance à la race blanche et qu'il appartient soit aux autorités soit aux tribunaux de se prononcer sur l'appartenance des individus à celle-ci. Bien après la fin de l'esclavage, les lois "Jim Crow" organisaient une ségrégation raciale dans le sud des États-Unis, qui séparaient Noirs et Blancs et écartaient les Noirs des urnes, l'appartenance des individus à l'une ou l'autre des catégories étant localement définie à partir de critères qui n'étaient pas seulement la couleur de la peau (16). Cet édifice n'est que tardivement et très progressivement démantelé. Cela favorise la naissance et la persistance de campagnes et d'organisations mobilisant, sur le terrain politique, autour de la défense des droits des Noirs et justifie la présence de catégories raciales et ethniques dans les recensements, dont la délimitation est dictée plus par les impératifs de l'action publique et le contexte politique que par des exigences scientifiques (17). L'historien étudiant l'histoire des populations noires fait alors aux États-Unis l'histoire des hommes et des femmes appartenant à un groupe qu'il n'a pas à construire, mais dont la composition lui est donnée par ses sources. Je ne vois guère comment l'historien de la France contemporaine pourra écrire une telle histoire sans utiliser l'origine ou l'hérédité comme indicateur, c'est-à-dire sans être ramené à une définition essentialiste du groupe.

En somme, et je suis là d'accord avec les prémisses posés par Pap Ndiaye, je considère, après bien d'autres, que le Noir n'existe pas mais que la qualité de noir qui n'est jamais la seule attachée à un individu, pensons à l'âge ou au genre - est attribuée à des individus dans certains contextes, ce qui a pour eux d'importants effets sociaux et pratiques - sans que cela implique pour autant que cette qualité soit toujours et partout la plus efficace de celles possédées par l'individu -, qu'une identité noire peut en naître et qu'il est possible d'en faire l'histoire, parce qu'il y a changement dans le temps de ces agencements sociaux, tant des modes d'attribution de cette qualité que de ses effets ou des stratégies et des identités des individus pris dans ces processus et que cette histoire est non seulement légitime mais aussi sans doute

(15) H. Le Bras, L'adieu aux masses. Démographie et politique, $2^{e}$ éd., Paris, L'Aube, 2005.

(16) C. W. WoodWARD, The strange career of Jim Crow, Oxford, Oxford University Press, $3^{e}$ éd. 1974.

(17) P. SCHOR, "Mobilising for pure prestige? Challenging Federal census ethnic categories in the USA (1850-1940) ", International Social Science Journal, March 2005, p. 89-101. 
aujourd'hui nécessaire. Je considère en revanche que l'on ne peut a priori déduire de cela l'existence et la pérennité d'un groupe social, si l'on donne un sens fort à ce terme, et que le vocabulaire de la démographie est peu compatible avec cet agenda constructiviste. Même si de tels propos peuvent sembler quelque peu ésotériques, puisque nous discutons ici de concepts guidant des travaux qui sont à écrire, il me semble qu'il ne s'agit pas simplement de pureté lexicale, ni de raffinements conceptuels. D'une part les enjeux idéologiques et donc pratiques de tels débats, et Pap Ndiaye le rappelle à plusieurs reprises, ne sont pas minces et je redoute sans doute plus que lui que ne s'imposent comme produit d'un découpage "naturel " du social, validées par l'appareil d'État et une partie de la communauté scientifique, des catégories renvoyant à la race et à l'origine, dont les usages pratiques ne seraient pas nécessairement ceux prévus par les précautionneux statisticiens les mettant au point. D'autre part, les catégories et le lexique posés par l'enquêteur ne sont pas sans effet sur le choix des sources et des dispositifs d'enquête et donc des conclusions.

Reste la question de la spécificité des discriminations frappant les individus en tant que Noirs et de ses effets, au nombre desquels nous pouvons compter, dans la perspective adoptée par l'auteur, les contours des identités noires. Elle est tranchée par Pap Ndiaye, dont l'argument est que le facteur mélanique ne permet pas de jouer avec les identités prescrites. L'argument à mon sens mériterait d'être examiné plus longuement. Il n'est pas certain, en l'absence pourtant d'un facteur mélanique, que les Turcs d'Allemagne, longtemps peu nombreux, même quand nés en Allemagne, à pouvoir prétendre à la citoyenneté allemande, aient eu beaucoup plus de marges de manœuvre (18), du fait de la puissance des identités de papier dans les sociétés contemporaines. De plus, l'identité noire n'est pas la seule identité sociale, corporellement inscrite, sur laquelle aient été fondées des discriminations : les Nippobrésiliens en fournissent un exemple dans un autre contexte, de même, dans le contexte français, que les identités de genre, et l'on peut se demander si l'expérience sociale - tant en ses effets pratiques qu'en la façon dont elle affecte l'image de soi de jeunes hommes de la classe moyenne se heurtant, parce que définis comme noirs, à un plafond de verre n'est pas plus proche de celles de leurs collègues de sexe féminin que de celles de clandestins renvoyés en Afrique par la voie des airs. Surtout, il me semble que mettre en avant la recherche de la spécificité des discriminations subies et des réponses à celle-ci par la mise en chantier d'une histoire dont l'objet serait les "Noirs de France" (19), affirmée comme distincte d'une histoire générale, elle aussi à venir, des discriminations ethnoraciales dans le contexte français, conduit à une aporie car "limiter et resserrer l'étude à une seule société pour dégager le Zusammenhang social est justement se condamner d'avance à ne jamais l'établir [...]. Le cas unique n'a pas de cause, n'est pas scientifiquement explicable " (20). Nous dirions aujourd'hui que la spécificité d'un phénomène n'a de sens que si celui-ci

(18) C. LEGGEWIE, "Turcs, Kurdes et Allemands. Histoire d'une migration : de la stratification sociale à la différenciation culturelle, 1961-1990 ", Le Mouvement Social, juillet-septembre 1999, p. 103-118.

(19) P. NDIAYE, "Pour une histoire des populations... ", art. cit., p. 108.

(20) F. SIMIAND, "Méthode historique et science sociale", Revue de Synthèse historique, 1903, p. 136 . 
PHILIPPE RYGIEL

est rapporté et comparé à une collection d'objets similaires, sous peine de retrouver en conclusion une spécificité d'emblée posée comme telle et forcément rapportée à ce qui fait l'unicité de la société ou du destin historique du groupe étudié.

Ces réserves ne doivent pas masquer le fait qu'une histoire des usages sociaux et politiques de la race dans le domaine français, qui intègre l'expérience et l'action de ceux qui eurent à en subir les conséquences, reste à faire, et je ne doute pas d'ailleurs que les ouvrages de Pap Ndiaye y contribueront, même si je préférerais à une histoire des populations noires de France une histoire de la fabrication des Noirs en France qui ne s'attache pas seulement aux spécificités de celle-ci. 